\title{
Sound speed optimization based on fuzzy sets using image texture as quality factors
}

\author{
Xingwu $\mathrm{He}$ \\ Network \& Information Management Center, Chengdu Normal University, Chengdu, Sichuan, China
}

\begin{abstract}
A constant sound speed of $1540 \mathrm{~m} / \mathrm{s}$ is usually used by ultrasound imaging systems for calculating delay curves in beamforming. A mismatch of this constant system sound speed and the real one in human tissue could result in significant defocusing and degrade the image resolution in the modern ultrasound imaging system. This paper has investigated characteristics and potential relationship of image texture parameters to image focusing quality due to unmatched speed of sound. Algorithms for sound speed optimization (SSO) system based on fuzzy process and image texture parameters are presented. The proposed method has been investigated and verified from phantom imaging including speckle, point targets and cyst images. Test results show that individual texture feature may help to determine appropriate sound speed used for beamforming; however, only the proposed fuzzy process based system can achieve the best sound speed detection with consistent and robust output.
\end{abstract}

\section{Introduction}

In medical ultrasound imaging systems, the sound speed is an important parameter for beamforming. It is usually set as a constant in human tissue, typically $1540 \mathrm{~m} / \mathrm{s}$ and his parameter is used to calculate electronic time delay [1]. These delay curves ensure that the ultrasound signals of all the array elements are in phase at the focal point. The actual propagation velocity varies over a very large range in human tissues, for instance, $1450 \mathrm{~m} / \mathrm{s}$ in fat and $1560 \mathrm{~m} / \mathrm{s}$ in kidney [2]; moreover, the same tissue may have different sound speeds in different patients.

The mismatched sound speed would lead to a shift of structure position, widening the point spread function and increasing side lobe levels of the image and then degrades image detail and contrast resolution [3]. Therefore, finding a way to select the best global sound speed used in system's delay profiles could be seen as a first-order phase aberration correction method. Zhao and Trahey [4] developed a method which could correct phase aberration by maximizing either mean brightness of diffuse or point targets. Napolitano et. al. [5] described an algorithm to find out the sound speed that produces the best overall lateral image quality by analyzing the spatial frequency content of the envelope data using various trial sound speeds. He and Liu [6] developed methods with raw radio frequency (RF) data and experimental results show more accurate sound speed than image based methods, while it needs much more computational requirement. Due to the shift of the focusing away from its theoretical acoustic focusing caused by the mismatched sound speed, Li and Liu [7] proposed a method to find the best sound speed by one of image texture parameters.

On account of significant defocusing and some geometric distortions leading to the effect of aberration detected in the reconstructed image as opposed to the aperture data. Authors [24] proposed 
an image registration-based sound speed estimation method that can be implemented using two images with different steer angles. However, for accurate registration, good image qualities in terms of detail and contrast resolution, as well as sophisticated registration procedures are all necessary. Moreover, the varying local echo phase when changing the transmit beam steering angle directly reflects the varying arrival time of the transmit wave front. Michael Jaeger [21] have described a method by sensing the angle-dependent aberration delay in a spatially resolved way and thus aberration correction for a spatially distributed volume aberrator, which achieved location-independent diffraction-limited resolution as well as accurate display of echo location based on reconstructing the speed-of-sound spatially resolved in phantoms. Clinical research in [22] shows sound speed correction can be used as an initial diagnosis in the disagree of homogeneous fatty liver. Guenther [23] proposed a method based on a modified inverse Radon transform for speed-of-sound correction, and the reconstruction using the modified inverse Radon transform shows a small but distinguishable improvement.

Quantitative results from [8] show that the sound speed error degrades the contrast of the point target, while the texture of the speckle does not appear significantly changed. Meanwhile, isolated point reflectors couldn't be present in clinical ultrasound imaging [9]. Therefore, to deal with complex clinical cases, this paper presents a sound speed optimization system based on fuzzy sets with image texture as quality factors. It not only integrated the sound speed correction method proposed by [4] and the focusing quality factor based on image texture parameters described by [7] but also built a decision-making system with fuzzy logic in order to make the texture analysis be more robust. This is a practical approach for sound speed optimization. Our experimental results on the phantom proved that this sound speed optimization system is accurate and robust on simulated tissue for speckle, focusing (from point targets) and cyst.

\section{Method}

\subsection{Image texture as quality factors}

Image texture has been used as a tool in image analysis; in [11], there are nine commonly used parameters for texture classification. In this paper, we classify them into four groups with properties related to image quality.

Group 1 (mean): represents the intensity of local region. The better the beam focusing, the greater it is.

Group 2 (variance, correlation, cluster shade and cluster prominence): describes the degree of image difference or linearity. Since the defocusing from mismatched sound speed wouldn't definitely produce the change of image difference or linearity for nonlinear tissue structures; and from our experiments, all of them are not quite associated with the focus quality. Thus, this paper doesn't choose any of them as the quality factors.

Group 3 (energy, entropy and homogeneity): measures homogeneity related texture. The more homogeneous the image is, the larger the value of energy/homogeneity is. Comparing to texture homogeneity, texture energy is more sensitive and directly to the focusing quality. While the entropy is in inverse to the energy, one can choose either of them as the quality factor. In this paper, the texture energy is used.

Group 4 (contrast): depicts local image variations. In general, local image with clear border/structure always shows larger texture contrast.

Then texture features used in this paper as quality factors are energy, contrast and mean as follows [11]:

Energy:

$$
\sum_{i} P_{S}(i)^{2} \sum_{j} P_{d}(j)^{2}
$$


Contrast:

$$
\sum_{j} j^{2} P_{d}(j)
$$

Mean:

$$
\frac{1}{2} \sum_{i} i P_{s}(i)
$$

where $P_{s}$ and $P_{d}$ are defined respectively by

$$
P_{s}\left(i ; d_{x}, d_{y}\right)=\operatorname{Card}\left\{(k, l) \mid y_{k, l}+y_{k+d_{x}, l+d_{y}}=i\right\}
$$

and

$$
P_{d}\left(i ; d_{x}, d_{y}\right)=\operatorname{Card}\left\{(k, l) \mid y_{k, l}-y_{k+d_{x}, l+d_{y}}=i\right\}
$$

In Eqs. (4) and (5), Card refers to the number of elements of a set $(k, l)$ and $y_{k, l}=j$ means that the intensity at the position $(k, l)$ is equal to the grey level of $j$. Notation $\left(d_{x}, d_{y}\right)$ denotes the direction vector along $x$ and $y$ direction, respectively. In this paper, we compute texture parameters of Eqs. (1), (2) and (3) along lateral direction (i.e., perpendicular to ultrasound beam direction) because the ultrasound speckle always shows longer size in lateral compared with that in axial direction [12].

\subsection{The fuzzy system model of sound speed optimization}

To improve the decision-making results of texture analysis for sound speed prediction, we applied the concept of the fuzzy sets because fuzzy logic can be used to address the issues associated with intrinsic imprecision or inexactness [13]. We define the fuzzy sets from the relationships between the selected image textures and image quality derived from a set of images with trial sound speed values. Mismatched speed of sound would produce a shift in axial direction which will involve confusion regions in ultrasound image; especially the decimation of pulse-echo signal for display would exaggerate the degradation of detail resolution. And in lateral direction, the mis-focus makes the effective pulse-echo beam width become wider, it means the edge of tissue structure in ultrasound image will be blurred and the contrast will be also reduced. It means the larger texture contrast is related to the better focused image. The simulation results in [20] shows it will be broadening in terms of the mis-focus target due to the error of assumed sound speed, which is consistent with lower texture energy. And better focused image leads to larger local image intensity that can be represented by larger texture mean. Based on the characteristics of focusing image, we can have a basic principle to judge a sample corresponding to an acceptable sound speed value:

"If contrast is LARGE, mean is LARGE, and energy is SMALL, then the focusing quality is GOOD.".

The basic linguistic rules can be used to do the approximate reasoning with suitable linguistic variables and these variables are described as follows:

Let $F$ be the linguistic variable for focusing quality with the universe of discourse (UoD) $U=$ $\{$ contrast, energy, mean $\}=\{\mathrm{u} 1, \mathrm{u} 2, \mathrm{u} 3\}$. The terms of this linguistic variable are set to be:

$\mathrm{T}(\mathrm{F})=\{$ VERY GOOD (VG), GOOD (G), FAIR (F), POOR (P), VERY POOR (VP) $\}$.

Also, the UoD of contrast, energy and mean are defined as $U C=\{\mathrm{c} 1, \mathrm{c} 2 \ldots \mathrm{cn}\}, \mathrm{UE}=\{\mathrm{e} 1, \mathrm{e} 2 \ldots$ en $\}$ and $U M=\{m 1, m 2 \ldots m n\}$, where ci, ei, and mi are values of the contrast, energy and mean of the ith images with a trial sound speed, respectively. The terms of these linguistic variables are all set to be:

$\mathrm{T}(\mathrm{C}|\mathrm{E}| \mathrm{M})=\{$ LARGE, MEDIUM, SMALL $\}$. 
In this paper we collect the fuzzy rules as the fuzzy associative memory which should preserve the basic properties of the individual's qualitative preference structure [14], as shown in Table 1. As we defined T (F), the linguistic variable in this section including P, VP as the terms, however, the fuzzy rules in Table 1 don't cover them because the decision can't be the one that produced the image with poor focusing quality.

Table 1. Fuzzy rules

\begin{tabular}{|c|c|c|c|c||c|c|c|c|c|}
\hline Rules & Energy & Contrast & Mean & Focusing quality & Rules & Energy & Contrast & Mean & Focusing quality \\
\hline 1 & small & large & large & very good & 7 & medium & large & small & fair \\
\hline 2 & medium & large & large & good & 8 & large & large & medium & fair \\
\hline 3 & small & large & medium & good & 9 & small & large & small & fair \\
\hline 4 & small & medium & large & good & 10 & small & medium & medium & fair \\
\hline 5 & medium & large & medium & good & 11 & medium & medium & large & fair \\
\hline 6 & larger & large & large & fair & & & & & \\
\hline
\end{tabular}

The membership functions used in fuzzy processing, in general, should meet the concept of optimal interfaces defined in [15]. The triangular membership function overlapping at a degree of 0.5 satisfies the concept of optimal interfaces in the sense of an error-free conversion between the numeric and the linguistic terms. Each membership function is defined by its centers, hereafter denoted by a tuple X, e.g. $\mathrm{X}(\mathrm{X} 0, \mathrm{X} 1, \mathrm{X} 2)$ for contrast, energy or mean; and $\mathrm{Xc}(\mathrm{X} 0, \mathrm{X} 1, \mathrm{X} 2)$ means the centers of each terms for contrast. The choice of these threshold vectors won't change the partial order in decision group. Approximate reasoning is a type of reasoning which is neither very exact nor very inexact [16]. Our general framework for reasoning is based on a linguistic model as follows:

Using the logical formula for "and" (intersection) in [17], see Eq. (6), and then obtain the approximate reasoning and decision-making function, see Eq. (7).

$$
\begin{gathered}
\mathrm{B}_{1} \text { and } \mathrm{B}_{2}=\mathrm{B}_{1} \wedge \mathrm{B}_{2}=\min \left\{\mathrm{B}_{1}, \mathrm{~B}_{2}\right\} \\
\mu_{D}(s)=\mu_{E}(s) \wedge \mu_{C}(s) \wedge \mu_{M}(s)
\end{gathered}
$$

where $\mu \mathrm{E}, \mu \mathrm{C}$ and $\mu \mathrm{M}$ are the degrees of membership in the term sets for the decision-making terms energy, contrast and mean, respectively, and is computed from the triangular membership function. In Eq. (7), S denotes a trial sound speed set, i.e., the decision group.

After producing all the $\mu \mathrm{E}, \mu \mathrm{C}$ and $\mu \mathrm{M}$ of each one in $\mathrm{S}$, we apply the fuzzy rules in Table 1 with Eq. (6). Then the degrees of membership of $\mathrm{VG}, \mathrm{G}$ and $\mathrm{F}$ sets in $\mathrm{T}(\mathrm{F})$ in $\mathrm{S}$ are determined by the max value of the approximate reasoning results for each term set, e.g. the one for $G$ is the max one in the reasoning results from Rules 2 to 5. Moreover, the decision for the best sound speed $\mathrm{s}^{*}$ is to find the $\max \mu \mathrm{D}$ in the term sets of focusing quality. It means that we first search the super $\mu \mathrm{D}$ in VG with the rule 1; if no one in that term set, then continue the search in $\mathrm{G}$, and then in F.

\subsection{The flowchart of the Sound Speed Optimization (SSO) system}

To simulate our sound speed optimization (SSO) techniques, we developed a computer program for the decision making with fuzzy sets, Figure1, the flowchart of the SSO system.

The beamformed I/Q data is the input for our simulation program. Firstly, each beamformed I/Q acoustic frame is square law and log detected but without scan conversion as the scan conversion will change the texture analysis consequences. Then, compute parameters of image textures in selected region of interest (ROI). The ROI should cover enough areas excluding the far and near field with better signal-to-noise signals and meaningful statistics. To get rid of signals from anechoic areas, the anechoic area is detected with pre-setting thresholds such as mean and variance. Then, the values of textures parameters must be normalized by the pre-setting extreme value in ROI. Next, the degrees of membership of each texture to the corresponding term sets in $T(C|E| M)$ are calculated by the standard triangular membership function [18] with the pre-setting tuple $\mathrm{X}$ on account of the examined part. After fuzzifying the texture data with the triangular and shouldered membership functions, we 
compute the degrees of membership of the term sets in $\mathrm{T}(\mathrm{F})$ following the approximate reasoning rules defined in Table I. That is, the degrees of membership about the focusing quality of ultrasound images produced by varied speeds of sound are decided. Finally, we search the sound speed $\mathrm{s}^{*}$ that produced the best focusing quality. It means we orderly check degrees of membership of the term sets in $\mathrm{T}(\mathrm{F})$ and finds the best one.

\section{Results}

This section will show the efficacy of the proposed SSO system with three typical cases from ultrasound phantom imaging. First, we take the speckle image as an example to show the relationships between nine image textures and speed of sound and verify the argument from our previous analysis. Then the performance of point targets and cyst images will be discussed.

Test data was acquired from Saset iMago C21 ultrasound scanner using SH5L38 linear array probe at $5 \mathrm{MHz}$ center frequency. Also, we used the CIRS Model 054 ultrasound phantom to acquire the speckle, point targets and cyst images. The speed of sound in this phantom is set to be $1544 \mathrm{~m} / \mathrm{s}$.

\subsection{Speckle image}

Figure 2.1 shows speckle images with a depth of $6 \mathrm{~cm}$ at a focal depth of $2.8 \mathrm{~cm}$. The ROI covers the area of the focal region with about 60 speckle resolution cells in axial direction $(0.35 \mathrm{~mm}$ per cell for $5 \mathrm{MHz}$ probe). The direction $(\mathrm{dx}, \mathrm{dy})$ for texture analysis is $(2,0)$ and the window size for texture computations is $16 \times 16$.

The normalized texture value in Figure 3 is the average of sample images with different speed of sound (15 sample images for each sound speed at the same location). From Figure 3, one can see that all textures except the group 2 show either peak or valley in the case of matched trial sound speed to the real one.

Three samples in one group of the speckle image are shown in Figure 2.1 where the speed of sound in the phantom is $1544 \mathrm{~m} / \mathrm{s}$. Using the unmatched system sound speed, the speckle image of Figs. 2.1.b and 2.1.c are worse than Figure 2.1.a in terms of speckle size around the focal region. This has been verified from the spectrum analysis described in [6]: the $-10 \mathrm{~dB}$ spectrum bandwidth for the $1400 \mathrm{~m} / \mathrm{s}$ (Figure 2.1.b) and $1700 \mathrm{~m} / \mathrm{s}$ (Figure 2.1.c) images are $84 \%$ and $92 \%$ of the correct one 1544 $\mathrm{m} / \mathrm{s}$ (Figure 2.1.a), respectively.

The performance of three selective textures on speckle image is shown in Figure 4.a as a function of trial sound speed. One can see that none of the extreme points of the contrast, mean and energy is located at $1544 \mathrm{~m} / \mathrm{s}$. Thus, it's not reliable to identify the best speed of sound with any one of them, though there are intrinsic relationships to the best detection in general.

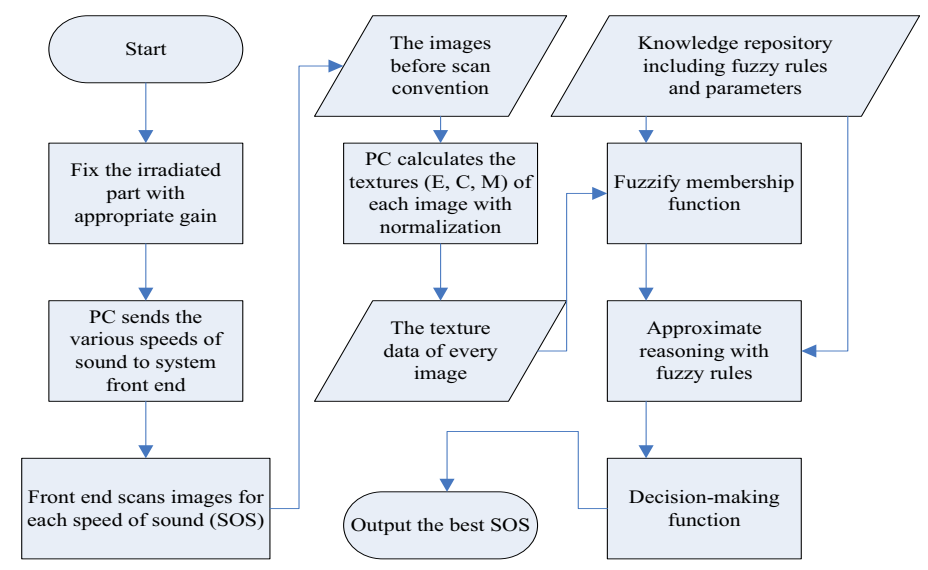

Figure 1. The flowchart of the sound speed optimization system 


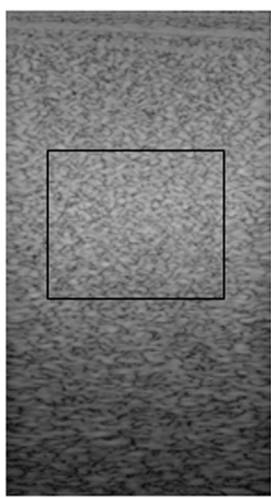

(2.1.a) $1544 \mathrm{~m} / \mathrm{s}$

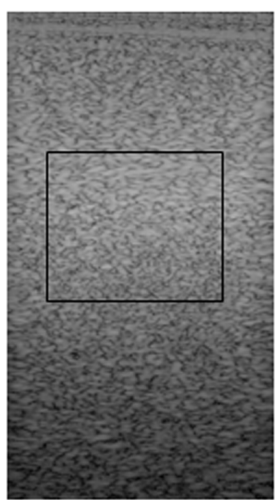

(2.1.b) $1400 \mathrm{~m} / \mathrm{s}(84 \%)$

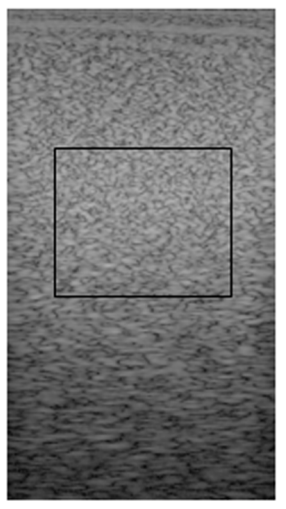

(2.1.c) $1700 \mathrm{~m} / \mathrm{s}(92 \%)$

Figurea 2.1. Speckle images with different preset sound speeds

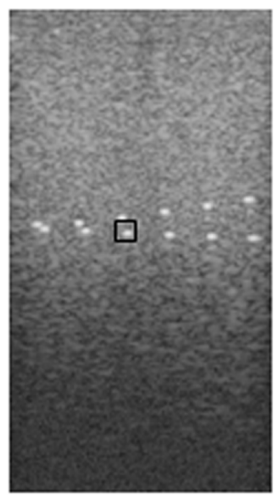

(2.2.a) $1544 \mathrm{~m} / \mathrm{s}$

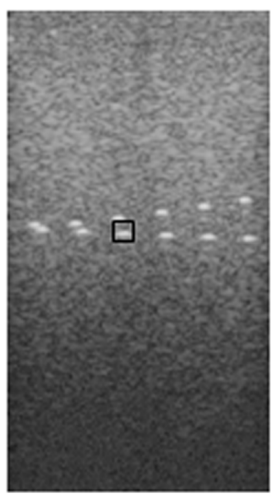

(2.2.b) $1400 \mathrm{~m} / \mathrm{s}(150 \%)$

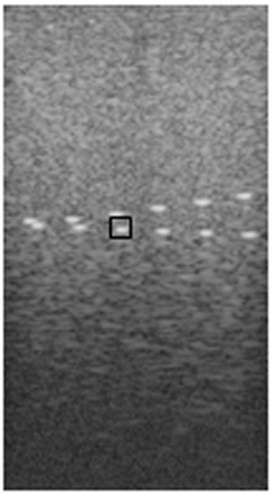

(2.2.c) $1700 \mathrm{~m} / \mathrm{s}(142 \%)$

Figure 2.2. Point target images with different preset sound speeds

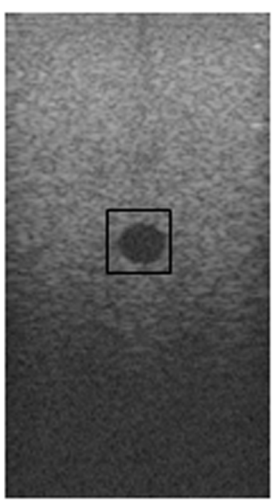

(2.3.a) $1544 \mathrm{~m} / \mathrm{s}(9.64)$

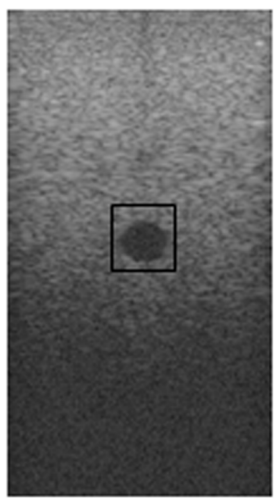

(2.3.b) $1400 \mathrm{~m} / \mathrm{s}(9.24)$

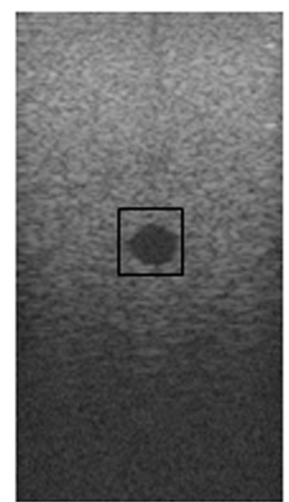

(2.3.c) $1700 \mathrm{~m} / \mathrm{s}(9.27)$

Figure 2.3. Cyst images with different preset sound speeds 


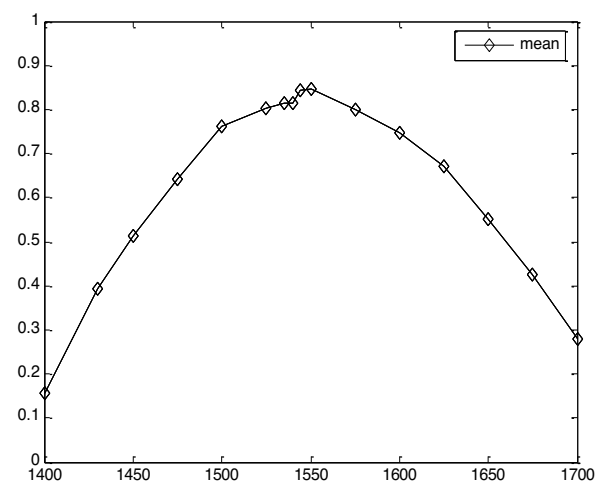

(3.a) Group 1

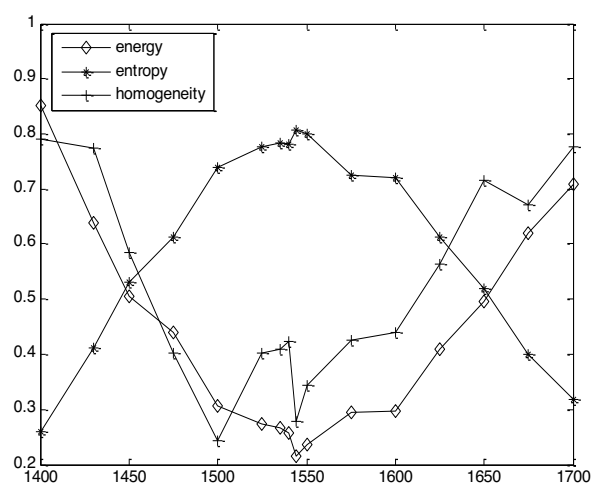

(3.c) Group 3

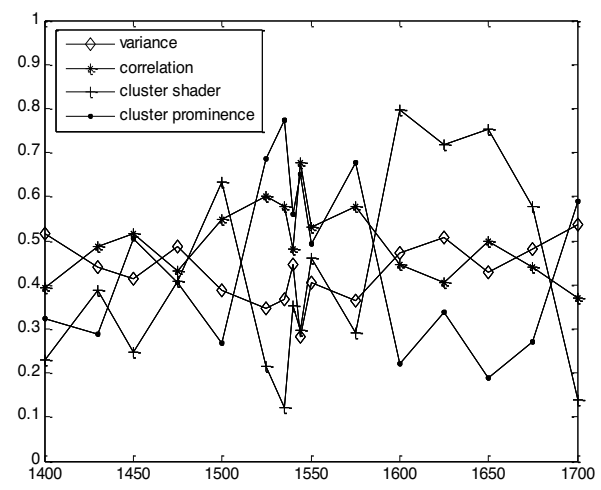

(3.b) Group 2

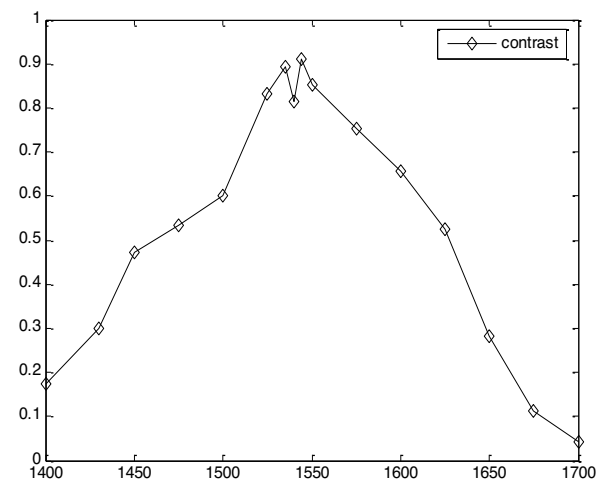

(3.d) Group 4

Figure 3. Nine texture features in four groups as mentioned in Section 2.1 on speckle image as a function of varied speed of sound.

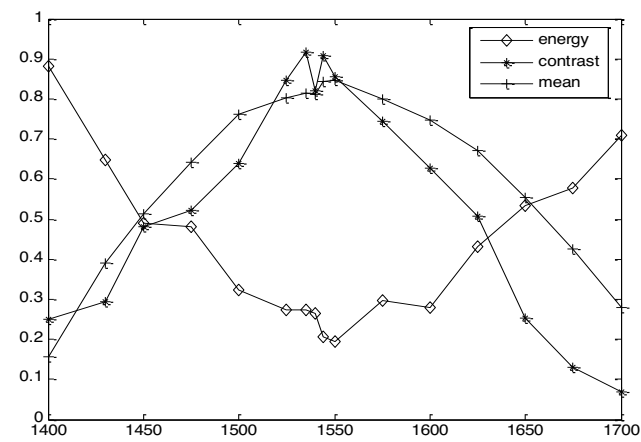

(4.a) speckle image

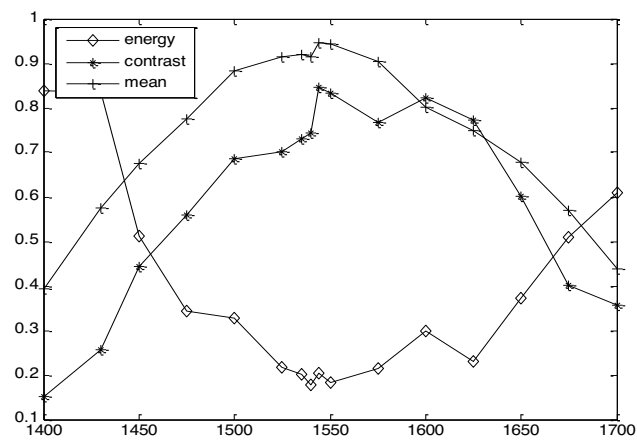

(4.b) point targets image 


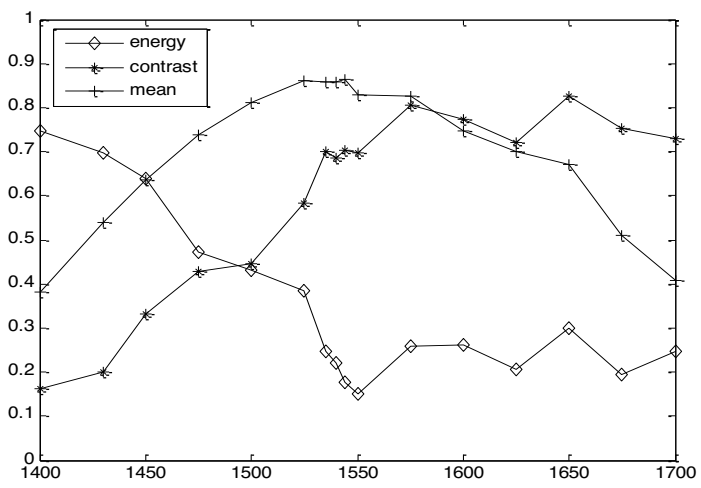

(4.c) cyst image

Figure 4. Three texture features on speckle, point targets and cyst images as a function of sound speed ( $\circ$ : Energy; *: Contrast; +: Mean).

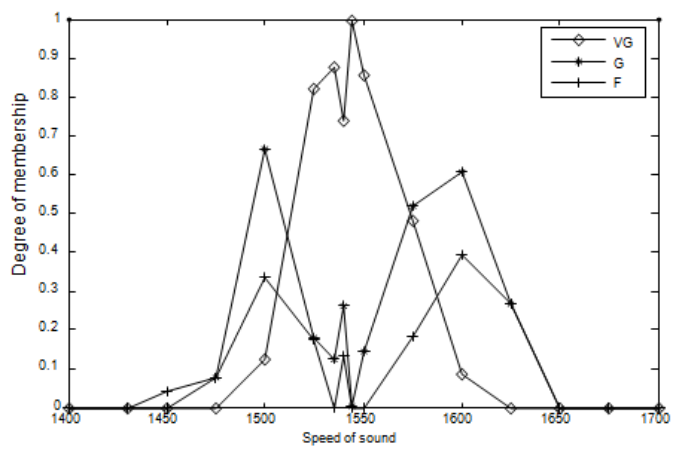

(5.a) speckle image

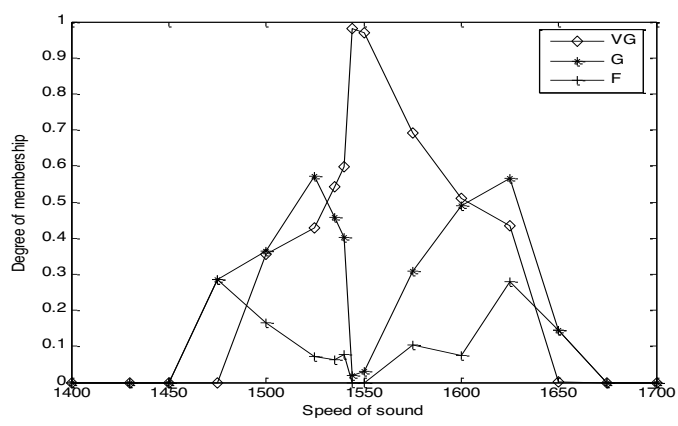

(5.b) point targets image

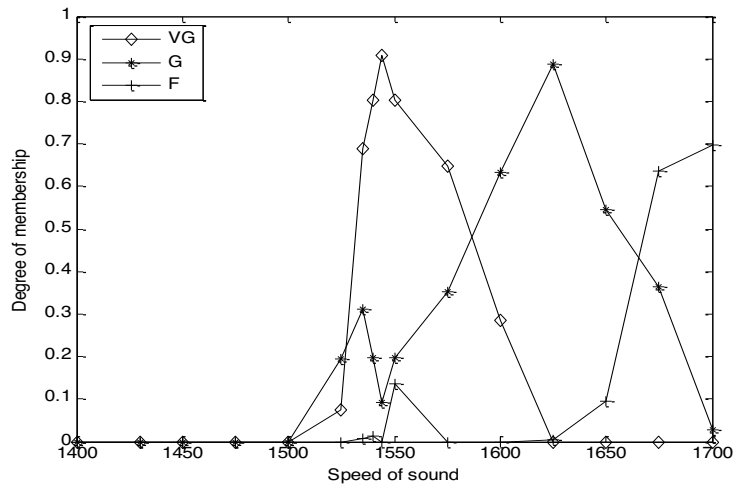

(5.c) cyst image

Figure 5. Outputs of the proposed SSO system with our fuzzy system $(\bigcirc:$ VG; *: G; +: F) 
From our fuzzy processing, we set the $\mathrm{XE}(0.24,0.5,0.7)$ (energy), $\mathrm{XC}(0.3,0.6,0.9)$ (contrast), $\mathrm{XM}(0.3,0.6,0.84)$ (mean) for the membership function of $\mathrm{T}(\mathrm{C}|\mathrm{E}| \mathrm{M})$ on the basis of the texture values and the principle of triangle overlaps between 10 to $50 \%$. Note that both the min and max of each texture value for normalization are fixed according to the location and the gain of imaging settings.

Figure 5.a shows the output of our SSO system where the y-axis represents the degrees of membership of every trial sound speed for each term set in $T(F)$. Here we list all the degrees of membership between the trial speed of sound and every term set in $\mathrm{T}(\mathrm{F})$. Following the previous description of our algorithm, we can find the best one in VG is $1544 \mathrm{~m} / \mathrm{s}$. Besides, we can decide the focusing quality of each trial sound speed by finding its maximum degree of membership.

\subsection{Point targets image}

The second group is obtained by the same way as the first group but there are several point targets in focal depth. Three samples of one group of the point targets images are shown in Figs. 2.2.a to 2.2.c. It's clear that the wrong system sound speeds of $1400 \mathrm{~m} / \mathrm{s}$ (Figure $2.2 . \mathrm{b}$ ) and $1700 \mathrm{~m} / \mathrm{s}$ (Figure 2.2.c) degrade the image quality in terms of broadening lateral resolution at point targets compared with the correct one of $1544 \mathrm{~m} / \mathrm{s}$ (Figure 2.2.a). The size of the point target in the rectangular area from $1400 \mathrm{~m} / \mathrm{s}$ and $1700 \mathrm{~m} / \mathrm{s}$ are increased by $50 \%$ and $42 \%$ respectively, compared to the one from $1544 \mathrm{~m} / \mathrm{s}$

Because of the point targets spreading, the peaks of contrast and mean of this group are both located at $1544 \mathrm{~m} / \mathrm{s}$, while the valley of energy is not; see Figure 4.b. Meanwhile, from the output of our SSO system, Figure 5.b with $\mathrm{XE}(0.2,0.4,0.7), \mathrm{XC}(0.3,0.6,0.85)$ and $\mathrm{XM}(0.3,0.6,0.92)$ for the membership functions of $\mathrm{T}(\mathrm{C}|\mathrm{E}| \mathrm{M})$, we find that the best one in VG is the correct one of $1544 \mathrm{~m} / \mathrm{s}$. Compared with the speckle image group, the broadening of the point targets due to unmatched sound speed, leads a significant decrease in detail and contrast resolution, while the texture of the speckle does not produce such a big change which meets with the results in [8].

\subsection{Cyst image}

The cyst images are also obtained by the same way as the above group but the cyst exists in focal depth. The region of texture analysis for this group covers about 50 speckle resolution cells in axial direction. Unlike point targets, the cyst shows both reflection and anechoic that is more realistic for tissue analysis. Three samples of the cyst images are shown in Figs. 2.3.a to 2.3.c. From image contrast-to-noise ratio (CNR),

$$
C N R=\frac{2\left(\mu_{t}-\mu_{b}\right)^{2}}{\sigma_{t}^{2}+\sigma_{b}^{2}}
$$

we can analyze the performance of our SSO system on cyst images. In Eq. (8) $\mu_{\mathrm{t}}$ and $\mu_{\mathrm{b}}$ denote the mean of target and background; and $\sigma_{t}$ and $\sigma_{b}$ are the standard deviation of target and background, respectively. A larger CNR helps better tissue differentiation from target (or tissue lesion) and image background.

In Figure 2.3, the CNR of the correct $1544 \mathrm{~m} / \mathrm{s}$ (Figure 2.3.a) in the rectangular box is 9.64 that is higher than the other two groups: 9.24 from $1400 \mathrm{~m} / \mathrm{s}$ (Figure $2.3 . \mathrm{b}$ ) and 9.27 from $1700 \mathrm{~m} / \mathrm{s}$ (Figure 2.3.c). It means that the image tissue differentiation degrades due to unmatched speed of sound. As a result of the intrinsic inexactness, the peak of contrast and also the mean and the valley of energy in this group, see Figure 4.c; fairly keep consistent to the focusing quality.

For our SSO system, we can find that the best speed of sound in VG is also accurate, $1544 \mathrm{~m} / \mathrm{s}$, and the distribution of $\mathrm{VG}$ is fairly consistent with the sound speed errors, see the output in Figure 5.c with $\mathrm{XE}(0.18,0.4,0.6), \mathrm{XC}(0.3,0.55,0.75)$ and $\mathrm{XM}(0.4,0.7,0.85)$ for the membership functions of $\mathrm{T}(\mathrm{C}$ | $\mathrm{E} \mid \mathrm{M})$. It shows that the capability of the fuzzy processing to make the decision is more robust, and also verifies our fuzzy rules to be feasible for different situations on phantom images. 


\section{Discussions}

From test results, the proposed SSO system could choose the accurate speed of sound based on a set of texture parameters. For the point target, it is clear that the sound speed error will cause the point targets broadening and lead to significant decrease in detail resolution. The off-axis response of the lateral point-spread-function (PSF) on speckle images also changes the texture mean. Unlike them, the texture energy does not depend on the brightness in ROI, but relate to the image homogeneity [19]. From point targets images, one can see the amplitude distribution of image pixels in the local window at the focal region doesn't change significantly, though the contrast and the mean brightness apparently changed with the sound speed errors. That's why texture energy shows less sensitive to the focusing quality of the point targets.

On the other hand, all of the selected textures have similar ability to depict the focusing quality in diffuse regions. Since contrast resolution and the homogeneity in local regions don't change significantly, none of them are completely sensitive to the sound speed errors. Compared with the texture contrast and energy, the texture mean is a little more sensitive which meets the theory in [4].

Unlike above twos, the sound speed errors won't make the cyst changed in terms of detail resolution. However, from CNR analysis, it seems that matched sound speed will help both background speckle and anechoic cyst inside with better mean brightness and clear cyst border in texture energy.

In this paper, we construct the fuzzy rules to do the approximate reasoning shown in Table 1. From this approach, we found that the status of each quality factor is not identical. Actually, the texture contrast has little preference for the group decision-making because of its higher sensitive to the sound speed errors which can be seen in [9]. So, not all the rules need to be used in actual processing, e.g. it is not necessary to perform the approximate reasoning when texture contrast definitely belongs to SMALL.

\section{Conclusion}

In this paper, a method of sound speed optimization based on fuzzy sets using image textures as focusing quality factors has been presented. The method is applicable to ultrasonic imaging system where the texture of the B-mode image is available. We investigated the properties of images textures with sound speed errors and built a decision-making system on the basic fuzzy rules. Results on phantom experiments for speckle, point targets and cyst image groups showed that the best sound speed from a set of images with trial speed of sound can be determined in our SSO system. From the sample images of each group, it's clear that the fuzzy process decision-making approach with texture based focusing quality factors behaves as a reliable and robust tool for optimal sound speed detection. Future works include in vivo tests and possible parameter optimization.

\section{Acknowledgements}

This study was supported by the project of Education Department of Sichuan Province (nos.16ZB0381).

\section{References}

1. Thomas L. Szabo, Diagnostic ultrasound imaging: inside out. 1st ed., 230-260 (Elsevier Academic Press, 2004)

2. F. A. Duck, Physical Properties of Tissues, 73-135 (Academic Press, London, 1990)

3. N.J. Dudley, N.M. Gibson, M.J. Fleckney, P.D. Clark, The effect of speed of sound in ultrasound test objects on lateral resolution, Ultrasound in Med. \& Biol, 28, 1561-1564 (2002) 
4. D. Zhao and G. E. Trahey, Comparisons of image quality factors for phase aberration correction with diffuse and point targets: theory and experiments, IEEE Transl. on Ultrasonics, Ferroelectrics, and Frequency control, 38 ,125-132 (1991)

5. D. Napolitano, C. H. Chou, et. al., Sound speed correction in ultrasound imaging, Ultrasonics, 44, 43-46 (2006)

6. Huanhuan He, Dong C. Liu, Sound Speed Optimization Based on Acoustic Point Spread Function, IEEE International Conference on Bioinformatics and Biomedical Engineering, 2112-2115 (2009)

7. Xiaoying Li, Dong C. Liu,An adaptive ultrasound sound speed optimization based on image contrast analysis, J. Biomedical Engineering, 30, 1094-1097 (2011)

8. M. E. Anderson, M.S. McKeag and G.E. Trahey, The impact of sound speed errors on medical ultrasound imaging, J. Acoust. Soc. Am, 107, 3540-3548 (2000)

9. S. W. Flax and M. O'Donnell, Phase-Aberration Correction Using Signals from Point Reflectors and Diffuse Scatters: Basic Principles, IEEE Transl. on Ultrasonics, Ferroelectrics, and Frequency control, 35,758-767 (1988)

10. Wen Liu, Yangjie Cheng and Dong C. Liu, Filter Based Receive-Side Spatial Compounding for Veterinary Ultrasound BMode Imaging, Bio-Medical Materials and Engineering, 24, 1193-1199 (2014)

11. M. Unser,Sum and difference histograms for texture classification, IEEE Trans. Pattern Anal. Mach. Intell., PAMI-8, 118-125 (1986)

12. J. W. Goodman, Some fundamental properties of speckle, Journal of Optical Society of America, 66, 1145-1150 (1976)

13. L. A. Zadeh, Fuzzy logic, Computer, 21, 83-93 (1988)

14. E. Cox, The Fuzzy Systems Handbook: A Practitioner's Guide to Building, Using, and Maintaining Fuzzy System, AP Professional, 340-600 (1994)

15. H.Tanaka,T.Okuda and K.Asai, Decision-making and its goal in a fuzzy environment, in:L.A.Zadeh,et al.(Eds.), Fuzzy Sets and Their Applications to Cgnitive and Decision Processes, 257-277 (Academic Press, 1975)

16. J.Valente de Oliveira, A design methodology for fuzzy system interfaces, IEEE Trans. Fuzzy Systems, 3, 404-414 (1995)

17. R. E. Bellman and L. A. Zadeh, decision-making in a fuzzy environment, Management science, 17, 141-164 (1970)

18. R. R. Yager and D. Basson, Decision making with fuzzy sets, Decision science, 6, 590-600 (1975)

19. Shuang Gao, Yuhua Peng, Huizhi Guo, Weifeng Liu, Tianxin Gao, Yuanqing Xu and Xiaoying Tang, Texture analysis and classification of ultrasound liver images, Bio-Medical Materials and Engineering, 24, 1209-1216 (2014)

20. Quan Chen and James A. Zagzebski, "Simulation study of effects of speed of sound and attenuation on ultrasound lateral resolution", Ultrasound in Med. \& Biol., 30, 1297-1306 (2004)

21. Michael Jaeger, Elise Robinson, H Gunhan Akarcay, Martin Frenz, "Full correction for spatially distributed speed-of-sound in echo ultrasound based on measuring aberration delays via transmit beam steering", Physics in Medicine and Biology, 60, 4497-4505 (2015)

22. Liu Jin-ying, Zhou Qi, Jiang Jue, Song Wei, "Application value of sound speed correction to estimating disagree of homogeneous fatty liver", Journal of Chinese Practical Diagnosis and Therapy, 5, 445-449 (2015)

23. Guenther Paltauf, Gerhild Wurzinger, Robert Nuster, "Speed-of-sound correction for photoacoustic and laser-ultrasound imaging with an integrating cylindrical detector", Proc. SPIE, Opto-Acoustic Methods and Applications in Biophotonics II, 16, 1117-1127 (2015)

24. J.F. Krücker, J.Brian Fowlkes, P.L.Carson, "Sound speed estimation using automatic ultrasound image registration", IEEE Translation on ultrasonics, ferroelectric, and frequency control, 51, 1095-1106 (2004) 